Zinin: Binwirkang des ïtherischen Senfols etc. 173

$$
\left.\mathrm{C}_{12} \mathrm{H}_{24} \mathrm{O}_{12}+4 \mathrm{C}_{8} \mathrm{H}_{8} \mathrm{O}_{3}-6 \text { aq. }=\text { Moringerbsāure }{ }^{*}\right)
$$

Gallussäure.

$" \quad+4 \mathrm{C}_{7} \mathrm{H}_{6} \mathrm{O}_{3}-6$ aq. = gewölınliche Gerbsăure. Rubichlorsäure.

$" \quad 4 \mathrm{C}_{7} \mathrm{H}_{10} \mathrm{O}_{5}-6 \mathrm{aq} .=$ Aspertannsāure ${ }^{* *}$ ).

Man sieht, dass die Kaffeesäıre, die Kalechusăure und Gallussäure homolog sind, eben so Rlodeoretinol und Saligenin.

Das. Chinaroth ist wahrscheinlich den andern rollen Substanzen, welche Gallussüure, Moringerbsüure etc. geben, älınlich.

\title{
XXIV.
}

\section{Ueber die Einwirkung des ätherischen Senföls auf die organischen Basen.}

Von

N. Zinin.

(Bullct. de St. Petersbourg.)

In allen künstlich dargestellten flüchtigen, organischen Basen hat sich, was das chemische Verhalten $2 u$ verschiedenen Körpern anbetrim, eine überraschende Analogie mit dem Ammoniak herausgestellt; - sie bilden nämlich Verbindungen, welche den Amiden und dem Harnstoffe entsprechen. Nicht ohne Interesse schien es mir, das Verhalten der organischen Basen, in Allgemeinen, zu dem ätherischen Senfol zu untersuchen, da bekanntlich Ammoniak mil demselben eine basische Verbindung einzugehen vermag. Bei diesen Vrrsuchen bat sich ergeben, dass die vorerwāhnte Analogie der flüchtigen künst-

und schwarzen Stoffe, wie der gewöhuliche ferbstoff giebt, ist es daher walırscheinlich, dass șie auch Zucker enthült.

") Ihre Zersetzung in der Hitze und durch Salpctersiture und Schwefelsiuure, in Phensäure and Oxyphensäure, erklärt sich leicht, wenn man annimmt, dass diese Gerbsăare eine Säure $\mathrm{C}_{8} \mathrm{H}_{8} \mathrm{O}_{5}$, der Gallussäare homolog, enthält.

**) Die Umwandlang wurde noch nicht beobachtet; aber man erhält aus derselben Losung Aspertannsānre, Rubichlorsäure und Zrcker. 
lichen Basen mit Ammoniak fortbesteht, mit dem Unterschiede jedoch, dass die entstehenden Verbindungen entreder niemals oder sehr selten basischer Natur zu sein scheinen; wenigstens sind sie nentral von den mcisten Basen, welche ich bis jetzt der Einwirkung des ätherischen Senfüls unterworfen habe. Im Nachstehenden gebe ich die vorläufige Beschreibung der Verbindungen mit Naphtalidin und Anilin.

Hat man zu einer wcingeistigen Autlüsung des Naphtalidin Senföl gegossen, so bcobachtet man nach einiger Zeit aul der Obertlāche und dem Boden des Gemisches die Bildung weisser, kleiner, flachnadeliger, strahlformig zu Halbkugeln vereinigter Krystalle, die bald eine starke Kruste in der Flüssigkeit erzeugen.

Nimml man ungeführ 8 Theile 90 p. C. Weingeist auf einen Theil Naphtalidin, so ist in dieser Kruste der grüsste Theil der Verbindung ausgeschieden. Aus $43 \mathrm{Th}$. Naphtalidin und $30 \mathrm{Th}$. Senfül hatten sich bei der ersten Krystallisation $51 \mathrm{Th}$., beim Einkochen der rückständigen Flüssigkeit auf die Hälfe ihres Volumens, noch $18 \mathrm{Th}$. der Verbindung auskrystallisirt. Dieselbe ist unlüslich in Wasser, lüst sich in geringer Mlonge in kaltem, in grüsserer Menge aber in kochendem, starken Weingeiste auf; in Aether ist sie schwer löslich; beim Erhitzen auf $130^{\circ} \mathrm{C}$. schmilzt sie zu einer klaren, farblosen Flüssigkeit, welcbe beim Abkühlen zu einer weissen, kürnig-krystallinischen Masse gesteht. Bei vorsichtiger Destillation geltt ein grosser Theil des Kürpers, ohne merkliche Zersetzung, in Form einer ülartigen, farblosen, oder leicht gelblichen flüssigkeit über, die bein abkühlen nur nach langem Stehen, in Folge der Bildung von kürnigen Krystallen, erstarrt. Behandlung mit Bleioxydbydrat entzieht diesem Körper seinen Schwefel. Das dabei erhaltene neue Product lüst sich leicht in Weingeist auf; aus der heissen, spirituossen AufJüsung krystallisirt feinkürnig ein weisser, seidenglänzender Kürper, aus der rückständigen Lơsung schlāgt Wasser eine geringe Quantitāt einer weichen, salbenartigen, mit den körnigen Krystallen noch vermischten Substanz aus, welche jedoch in Weingeist bedeutend Ioslicher ist, als die körnigen Krystalle.

Die Elementaranalysen der Verbindung des NaphtalidinSenföls gaben folgende Zahlen:

1) 69,72 p. C. C.; 6,00 p. C. H.;

2) 69,60 p. C. C. ; 5,90 p. C. H.; 
3) 12,98 p. C. S.;

4) 13,02 p. C. $S$;

welche der Formel $\mathrm{C}_{28} \mathrm{H}_{14} \mathrm{~N}_{3} \mathrm{~S}_{2}=\mathrm{C}_{20} \mathrm{H}_{9} \mathrm{~N}+\mathrm{C}_{8} \mathrm{H}_{3} \mathrm{NS}_{2}$ entsprechen. Die Verbindung scheint mit Säuren sich nicht zu vereiniguen: aus weingeistigen salz- oder schwefelsäurehaltigen Lösungen $\mathrm{kry}$ stallisirt sie ohne Verānderung; in wässriger, concentrirter Salzsäure löst sie sich sogar in der Hitze nicht mehr als in reinem Wasser auf. In Salpetersãure (1,36 spec. Gew.) löst sie sich bei behutsamem Erhitzen farblos auf, bald aber fängt die Reaclion unter heftiger Entwickelung rother Dämple an, wobei die Flüssigkeit gelb wird und beim Zugiessen von Wasser scheidet sich aus derselben ein harziger, gelber Körper aus. Kocht man aber die saure Auflosung bis zum Verschwinden der rothen Dämpfe, so giebt sie auf Zusalz ron Wasser ein orangegelbes Pulver, welches sich in Weingeist und Salpetersäure leicht, in kochendem Wasser schwerer lóst, beim Erhitzen auf Platinblech zu einer bräunlichrothen Flüssigkeit schmilzt, mit Verpuffung verbrennt und viel Kohle hinterlāsst.

Giesst man zu einer Lösung von $1 \mathrm{Th}$. Anilin in ungefähr $4 \mathrm{~Tb}$. 90. p. C. Weingeist eine dem Anilın beinahe àquivalente Quantităt ātherischen Senföls, so beginnt bald die Verbindung; die Flüssigkeit erwärmt sich bedeutend, der Geruch des Oels wird kaum bemerkbar und es bildet sich wāhrend der Abkühlung eine Masse von feinen, blättrigen Krystallen. Nimmt man aber eine weniger concentrirte Lossung von Anilin, so werden die Krystalle, welche vier- und sechsseitige Tafeln sind,-bis 4 Millim. lang und $1 / 2$ Mlllim. dick. Diese sind vollkommen geschmack-, geruch- und farblos, durchsichtig, in Wasser unløslich, leicht lusslich sogar in kaltem Weingeist und in Aether; sie schmelzen bei $95^{\circ}$ C. zu einer durchsichtigen, farblosen Flüssigkeit, welche beim Abkühlen zu einer strahlig-krystallinischen Masse erstarrt. Erhöht man aber die Temperatur bis zum Kochen der Flüssigkeit, so entwickelt sich ein dem Lauche auffallend ähnlictrer Geruch und die Flüssigkeit bleibt tarauf beim Abkühlen lange zähe ohne zu erstarren; bei der Destillation derselben bekommt man eine ölige Flüssigkeit von demselben knoblauchartigen Geruch, welche aber auf keine Weise zum Erstarren zu bringen ist. Durch Einwirkung des Bleioxydhydrats wird der Verbindung des Anilins mit Senfol ihr Schwefel entzogen; es entsteht dabei eine 
in Weingeist selir leicht lüsliche Verbindung, so dass die spirituüse von dem Schwefelblei abgegossene Flüssigkeit erst auf Zusalz von Wasser - bis eine bemerkbare Trübung sogar heim Kochen bleibt - beim abkühlen, lange, seidengläuzende, nadelrormige Krystalle giebt, dic auch in kochendem Wasser sich ein weniz lösen und beim Abkülılen desselben ausgeschieden werden. Wasser schlāgt aus der von den nadelförmigen Krystallen abgegossenen Flüssigkeit eine kleine Quantität eines harzigen, zähen, in Weingeist leicht löslichen, nicht krystallisirbaren und in Wasser fost unlöslichen Körpers nieder.

Die Elementaranalysen des Anilin-Senfüls ergaben folgende Zahlen :

1) 62,80 p. C. C.; 6,50 p. C. H.;

2) 62,83 p. C. C.; 6,42 p. C. II.;

3) 16,02 p. C. S.;

4) 16,30 p. C. S.;

welche der Formel $\mathrm{C}_{20} \mathrm{H}_{12} \mathrm{~N}_{2} \mathrm{~S}_{2}=\mathrm{C}_{12} \mathrm{H}_{7} \mathrm{O}+\mathrm{C}_{3} \mathrm{H}_{3} \mathrm{NS}_{2}$ entsprechen. Dieser Körper scheint sicls mit Säuren auch nicht zu verbinden, Jơst sich jedoch in concentrirter wässriger Salzsāure Jejcht beim Erhitzen auf; von Wasser aber wird er aus dieser Lüsung unverāndert abgeschieden. Aus spirituōsen, salz- oder schıvefelsäurehaltigen Lüsungen krystallisirt er ebenfalls unverändert. Von kalter Sulpetersāure von 1,36 spcc. Gew. wird er nicht angegriffen; beim vorsichtigen Elbitzen lüst er sich darin auf, wobei aber nach kurzer Zeit eine heftige Einwirkung unter Entwickelung von rothen Dämpfen erfolgt; die Flüssigkeit wird gelb und giebt beim Vermischen mit Wasser einen gelben harzigen Niederschlag.

In einer Reihe von Aufsaatzen hoffe ich die Eigenschaften, Zusammensetzungen und die bemerkenswerthen Zersetzungsproducte der Verbindungen des Senföls mit verschiedenen Classen organischer Basen miltheilen zu kőnnen; hier bemerke ich nur, dass mit Harnstoffen und mit natürlichen nicht nüchtigen organischen Alkaloiden, wie Chinin, Cinchonin, Morphin und Narkotin, bis jetzt keine Verbindungen nit dem Senföl durch Zusammengiessen alkoholischer Lòsungen und Erwärmen, zu Stande gebracht wurden; Schwefelkoblenstoff scheint ebenfalls auf diesc Basen nicht einwirken zu wollen. 
Schliesslich sei es mir vergönnt, noch einige auf meine früheren Arbeiten Bezug habende Worte hinzuzufügen.

Azobenzid verwandelt sich leicht in Benzidin, wenn man seine weingeislige Lősung mit schwefliger Säure behandelt; es schlägt sich dabei sogleich das fast unlösliche schwefelsaure Salz der Base nieder.

Das Azoxybenzid verwandelt sich leicht in Benzidin bei der Behandlung seiner weingeistigen Lơsung mit reducirenden Mitteln.

Es ist mir gelungen, das Semitiaphtalidin vollstăndig farb- los darzustellen, und zwar durch wiederholte abwechselnde Krystallisation aus Weingeist und.Wasser. Die langen nadelformigen Krystalle sind sehr glānzend, wenn sie aus Weingeist ausscheiden. Das schwefelsaure Salz dieser Base ist schwer lüslicher in Wasser, als die Base selbst; es krystallisirt in kleinen schuppigen Krystallen von sveisser Farbe. Bei der Analyse gab es 38,20 p. C. Schwefelsăure; die Formel

$$
\mathrm{C}_{10} \mathrm{H}_{5} \mathrm{~N}, \mathrm{SHO}_{4}
$$

verlangt 38,28 p. C. Die kupferrothe Färbung der Hase rührte von einer geringen Beimischung eines andern bräunlich-rothen Korpers her, der in Weingeist viel leichter loslich ist als das Seminaphtalidin. Aus wässriger Lơsung krystallisirt dieser färbende Stoff in ganz feinen matten Nadeln, welche beim Erhitzen in einem Glasrőhrchen erst zu einer rothen Flüssigkeit schmelzen, und dann unter theilweiser Zerselzung destilliren, aber nicht verpuffen. 